\title{
Fe VII lines in the spectrum of RR Telescopii
}

\author{
P. R. Young ${ }^{1}$, K. A. Berrington ${ }^{2}$, and A. Lobel $^{3}$ \\ ${ }^{1}$ CCLRC Rutherford Appleton Laboratory, Chilton, Didcot, Oxfordshire OX11 0QX, UK \\ e-mail: p.r.young@rl.ac.uk \\ 2 School of Science and Mathematics, Sheffield Hallam University, Sheffield S1 1WB, UK \\ 3 Smithsonian Astrophysical Observatory, 60 Garden Street, Cambridge, MA 02138, USA
}

Received 21 September 2004 / Accepted 15 November 2004

\begin{abstract}
Thirteen transitions within the ground $3 \mathrm{~d}^{2}$ configuration of Fe VII are identified in ultraviolet and optical spectra of the symbiotic star RR Telescopii obtained with the STIS instrument of the Hubble Space Telescope. The line fluxes are compared with theoretical data computed with the recent atomic data of Berrington et al., and high resolution optical spectra from $V L T /$ UVES are used to identify blends. Seven branching ratios are measured, with three in good agreement with theory and one affected by blending. The $\lambda 5277 / \lambda 4943$ branching ratio is discrepant by $>3 \sigma$, indicating errors in the atomic data for the $\lambda 5277$ line. A least-squares minimization scheme is used to simultaneously derive the temperature, $T$, and density, $N_{\mathrm{e}}$, of the RR Tel nebula, and the interstellar extinction, $E(B-V)$, towards RR Tel from the complete set of emission lines. The derived values are: $\log T / \mathrm{K}=4.50 \pm 0.23, \log N_{\mathrm{e}} / \mathrm{cm}^{-3}=7.25 \pm 0.05$, and $E(B-V) \leq 0.27$. The extinction is not well-constrained by the Fe VII lines, but is consistent with the more accurate value $E(B-V)=0.109_{-0.059}^{+0.052}$ derived here from the $\mathrm{Ne}$ V $\lambda 2974 / \lambda 1574$ ratio in the STIS spectrum. Large differences between the K. A. Berrington et al. electron excitation data and the earlier F. P. Keenan \& P. H. Norrington data-set are demonstrated, and the latter is shown to give worse agreement with observations.
\end{abstract}

Key words. stars: binaries: symbiotic - stars: individual: RR Telescopii - ultraviolet: stars - atomic data

\section{Introduction}

RR Telescopii is a slow symbiotic nova that underwent an outburst in 1944 and whose optical light curve is still fading. The nature of the system is not fully understood but is thought to consist of a late-type giant and a hot white dwarf of effective temperature around $142000 \mathrm{~K}$ (Jordan et al. 1994). The white dwarf illuminates the nebula around the system, and gives rise to an extraordinarily rich nebular emission line spectrum, that has long been a testing ground for atomic data.

Comparatively little atomic data is available for Fe VII, on account of the complexity of the ion (which has an open $d$ shell), and the fact that Fe VII gives rise to only weak emission lines from the Sun's atmosphere. However, nebular plasmas under illumination from a hot source ( $\gtrsim 100000 \mathrm{~K})$ exhibit strong emission lines at visible wavelengths from within the Fe VII $3 \mathrm{~d}^{2}$ ground configuration. The transitions offer excellent plasma diagnostic possibilities (e.g., Nussbaumer \& Storey 1982).

In the present work we compare theoretical line emissivities calculated from the atomic data of Berrington et al. (2000) for Fe VII with the observed RR Tel fluxes measured by the STIS instrument on board the Hubble Space Telescope (HST). These observations give almost simultaneous measurements of the optical and UV lines, and allow the Fe VII atomic model to be tested.

\section{Atomic data}

The atomic data of Berrington et al. (2000) were used to construct a model of the Fe VII ion for inclusion in the CHIANTI database ${ }^{1}$ (Dere et al. 1997; Young et al. 2003). The model consists of all 9 fine structure levels in the ground $3 \mathrm{~d}^{2}$ configuration of the ion. The Maxwellian-averaged collision strengths $(\Upsilon)$ for all 36 transitions amongst the 9 levels were fit with 5 point splines, as described in Dere et al. (1997), for inclusion in CHIANTI. The fits have an accuracy of $\leq 1.4 \%$ over the temperature range $4.3 \leq \log T \leq 6.0$. The radiative decay rates are from Berrington et al. (2000), however three of the values given in Table 3 of Berrington et al. (2000) are incorrect. The $1-4,1-7$ and 4-7 decay rates should be 0.372 , 0.0150 , and $0.182 \mathrm{~s}^{-1}$.

The previous model of Fe VII used in CHIANTI made use of electron collision data from Keenan \& Norrington (1987) and radiative decay rates from Nussbaumer \& Storey (1982). Large discrepancies are found with the data of Keenan \& Norrington (1987) as illustrated in Fig. 1 for four transitions

\footnotetext{
${ }^{1}$ http://www.chianti.rl.ac.uk
} 

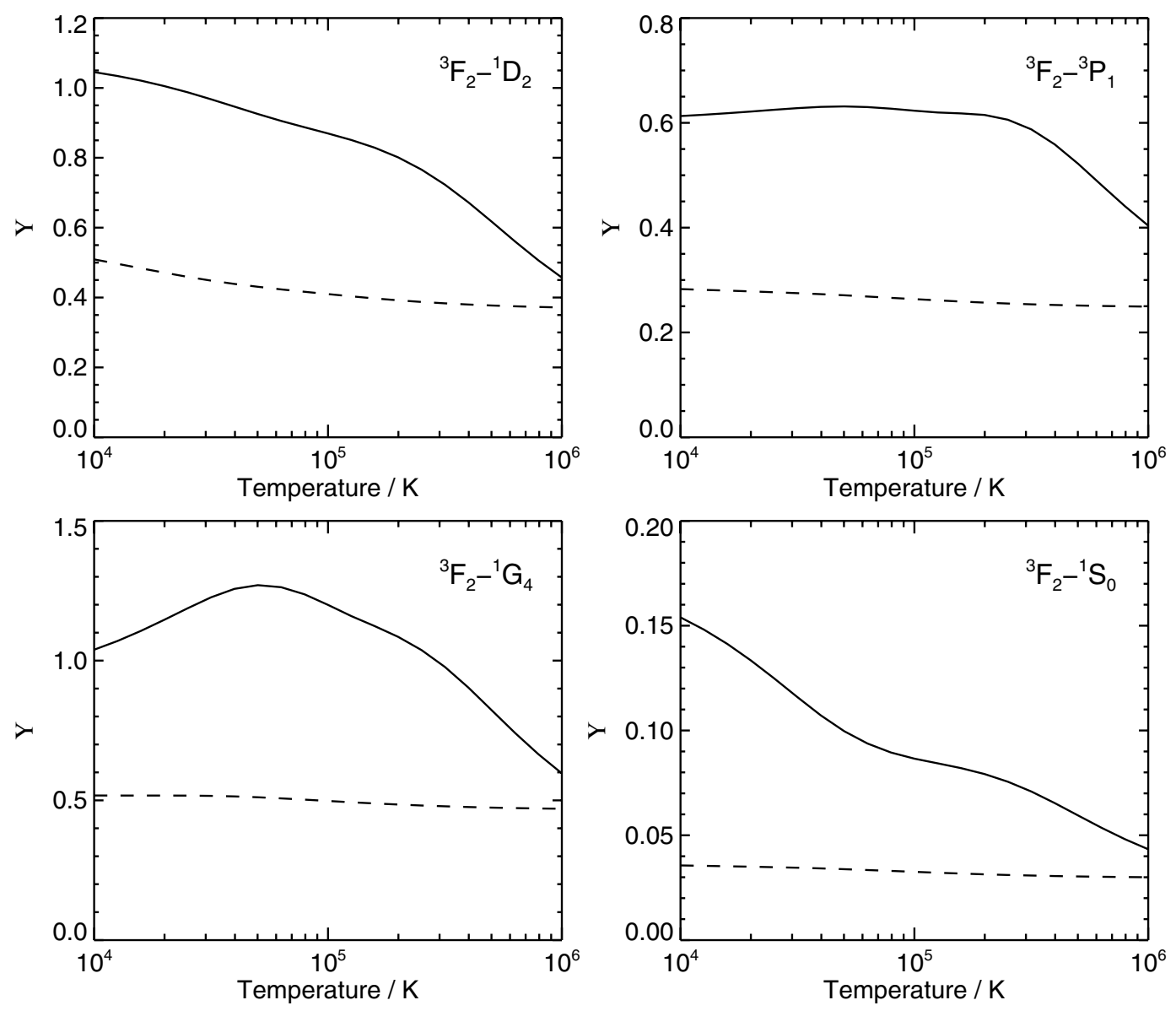

Fig. 1. Comparison of Maxwellian-averaged collision strengths ( $\Upsilon$ ) from Berrington et al. (2000) (solid line) with those of Keenan \& Norrington (1987) (dashed line) for four transitions of Fe VII. The values have been derived from the spline fits in CHIANTI. The original data cover $8000 \leq T \leq 120000 \mathrm{~K}$ (Keenan \& Norrington 1987) and $4.3 \leq \log T \leq 6.0$ (Berrington et al. 2000) and so values outside these ranges have been extrapolated.

from the ground ${ }^{3} \mathrm{~F}_{2}$ level. The differences are due to resonance structure not accounted for by Keenan \& Norrington (1987) see Berrington et al. (2000) for more details.

The radiative decay rates ( $A$-values) of Nussbaumer $\&$ Storey (1982) are in very good agreement with those of Berrington et al. (2000), as shown in Fig. 2. All but five of the $24 \mathrm{~A}$-values agree to within $30 \%$. The worst agreement is for the ${ }^{1} \mathrm{D}_{2}-{ }^{1} \mathrm{G}_{4}$ and ${ }^{3} \mathrm{P}_{2}-{ }^{1} \mathrm{G}_{4}$ transitions where the Berrington et al. (2000) values are factors 3.4 and 2.6 greater than those of Nussbaumer \& Storey (1982). Both transitions are weak, however, and the decays to the ${ }^{3} \mathrm{~F}_{3,4}$ are more important in depopulating the ${ }^{1} \mathrm{G}_{4}$ level.

Träbert et al. (2003) describe a laboratory measurement of the lifetime of the ${ }^{1} \mathrm{~S}_{0}$ level, giving $29.6 \pm 1.8 \mathrm{~ms}$. This compares very well with $28.7 \mathrm{~ms}$ from the Berrington et al. (2000) calculation. The Nussbaumer \& Storey (1982) calculations gave $33.1 \mathrm{~ms}$.

Software available in the CHIANTI database allows level populations and line emissivities to be calculated over a wide range of temperature and density (Dere et al. 1997). In Table 1, percentage level populations for the nine levels of Fe VII are given at two temperatures and three densities. $25000 \mathrm{~K}$ is a typical temperature for photoionized plasmas, while $250000 \mathrm{~K}$ is the temperature of maximum ionization of Fe VII in an electron ionized plasma.

\section{Observations}

RR Tel was observed on 2000 October 18 with the STIS instrument on HST. Complete spectra were obtained over the wavelength range $1140-7051 \AA$ in 3 HST orbits. The UV spectra (1140-3120 ̊) were obtained at a spectral resolution of around 30-45000, while the visible spectra (3022-7051 $\AA$ ) were obtained at a resolution of 5-10000.

STIS data are processed automatically with the most recent version of the STIS calibration pipeline when accessed through the $\mathrm{MAST}^{2}$ archive. 1D spectra are provided with fluxes and flux errors. Absolute fluxes are accurate to 5\% (CCD spectra) and 8\% (MAMA spectra); absolute wavelengths are accurate to $3-15 \mathrm{~km} \mathrm{~s}^{-1}$ (CCD spectra) and $1.5-5.0 \mathrm{~km} \mathrm{~s}^{-1}$ (MAMA spectra).

The Fe VII lines are exceptionally strong in RR Tel, and 13 lines can be identified, which are listed in Table 2. The lines were fit with Gaussian profiles through $\chi^{2}$ minimization

\footnotetext{
${ }^{2}$ Multimission Archive at Space Telescope,

http://archive.stsci.edu
} 
Table 1. Percentage level populations for Fe VII calculated at two values of the electron temperature and three values of the electron density $\left(N_{\mathrm{e}}=10^{6}, 10^{8}, 10^{10} \mathrm{~cm}^{-3}\right)$.

\begin{tabular}{llrrrrrrr}
\hline \hline & & \multicolumn{3}{c}{$25000 \mathrm{~K}$} & & \multicolumn{3}{c}{$250000 \mathrm{~K}$} \\
\cline { 3 - 5 } \cline { 7 - 9 } Index & Level & $10^{6}$ & $10^{8}$ & $10^{10}$ & & $10^{6}$ & $10^{8}$ & $10^{10}$ \\
\hline 1 & ${ }^{3} \mathrm{~F}_{2}$ & 43.05 & 20.29 & 19.45 & & 62.04 & 14.85 & 12.06 \\
2 & ${ }^{3} \mathrm{~F}_{3}$ & 34.60 & 26.79 & 25.63 & & 26.19 & 21.87 & 16.79 \\
3 & ${ }^{3} \mathrm{~F}_{4}$ & 19.69 & 31.12 & 30.60 & & 9.16 & 24.62 & 21.39 \\
4 & ${ }^{1} \mathrm{D}_{2}$ & 0.56 & 6.31 & 7.10 & & 0.40 & 8.70 & 10.93 \\
5 & ${ }^{3} \mathrm{P}_{0}$ & 0.37 & 1.17 & 1.23 & & 0.46 & 2.05 & 2.14 \\
6 & ${ }^{3} \mathrm{P}_{1}$ & 0.81 & 3.43 & 3.60 & & 0.88 & 6.24 & 6.43 \\
7 & ${ }^{3} \mathrm{P}_{2}$ & 0.64 & 5.30 & 5.71 & & 0.52 & 9.40 & 10.62 \\
8 & ${ }^{1} \mathrm{G}_{4}$ & 0.30 & 5.57 & 6.61 & & 0.35 & 12.15 & 18.20 \\
9 & ${ }^{1} \mathrm{~S}_{0}$ & $9.82(-5)$ & 0.02 & 0.08 & & $5.45(-4)$ & 0.10 & 1.44 \\
\hline
\end{tabular}

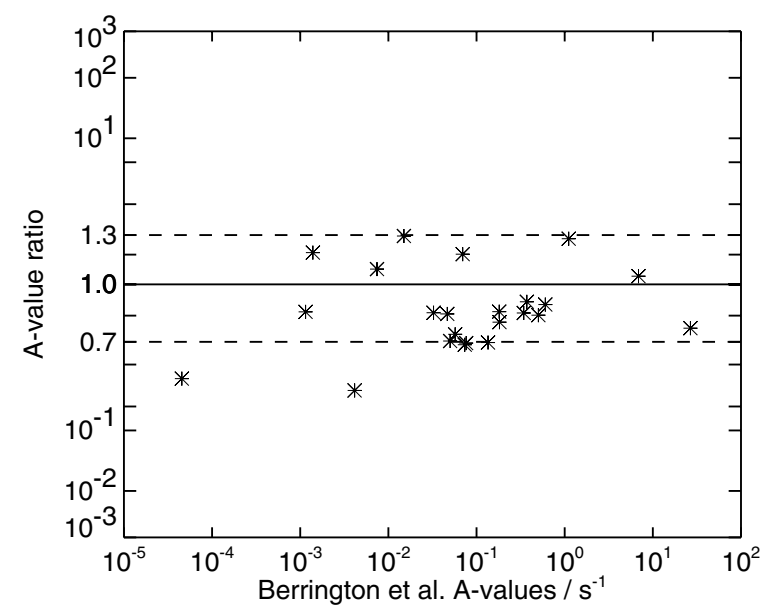

Fig. 2. Comparison of $A$-values from Berrington et al. (2000) with those of Nussbaumer \& Storey (1982). The $Y$-axis gives the ratio of the Nussbaumer \& Storey (1982) value to the Berrington et al. (2000) for a particular transition, while the $X$-axis value gives the Berrington et al. (2000) value. The $Y$-axis is scaled as the square root of the logarithm (base 10) of the ratio, allowing a wide range of values to be displayed. The solid line indicates perfect agreement (ratio $=1)$, and the dashed lines are placed at $\pm 30 \%$.

using the MPFIT curve fitting tools of C. B. Markwadt ${ }^{3}$ as distributed through Solarsoft ${ }^{4}$. For 11 of the 13 lines, fitting errors were smaller than the accuracy of the STIS absolute calibration, and so the line fluxes are accurate to 5 and $8 \%$ for lines in the CCD and MAMA spectra, respectively. The weak $\lambda 2183$ line has a significant fitting error, and the flux is $2.4 \pm 0.4 \times 10^{-14} \mathrm{erg} \mathrm{cm}^{-2} \mathrm{~s}^{-1}$. The Fe VII $\lambda 2143$ line is blended with N II $\lambda 2143.452$. The nearby N II $\lambda 2139.687$ line shares a common upper level with the latter, and the branching ratio $\lambda 2139 / \lambda 2143$ is 0.406 . The $\lambda 2139$ flux is $7.87 \times$ $10^{-14} \mathrm{erg} \mathrm{cm}^{-2} \mathrm{~s}^{-1}$, and by correcting for the branching ratio and subtracting this from the blended feature's flux, we

\footnotetext{
${ }^{3}$ http://astrog.physics.wisc. edu/ craigm/idl/idl .html

4 Solarsoft is a set of integrated software libraries, databases, and system utilities that provide a common programming and data analysis environment for Solar Physics. It is available at http://www. lmsal. com/solarsoft
}

Table 2. Fe VII line list for RR Telescopii.

\begin{tabular}{lrll}
\hline \hline$\lambda_{\text {meas }}(\AA)$ & Flux $^{a}$ & $\lambda_{\text {vac }}(\AA)$ & Transition \\
\hline 2015.570 & 67.4 & 2016.015 & ${ }^{1} \mathrm{D}_{2}-{ }^{1} \mathrm{~S}_{0}$ \\
$2143.085^{b}$ & 38.8 & 2143.706 & ${ }^{3} \mathrm{P}_{1}-{ }^{1} \mathrm{~S}_{0}$ \\
2182.902 & 2.4 & 2183.421 & ${ }^{3} \mathrm{P}_{2}-{ }^{1} \mathrm{~S}_{0}$ \\
3586.830 & 555.6 & 3587.341 & ${ }^{3} \mathrm{~F}_{3}-{ }^{1} \mathrm{G}_{4}$ \\
3759.526 & 838.0 & 3759.992 & ${ }^{3} \mathrm{~F}_{4}-{ }^{1} \mathrm{G}_{4}$ \\
4699.118 & 31.5 & 4699.557 & ${ }^{3} \mathrm{~F}_{2}-{ }^{3} \mathrm{P}_{2}$ \\
4893.933 & 58.5 & 4894.739 & ${ }^{3} \mathrm{~F}_{2}-{ }^{3} \mathrm{P}_{1}$ \\
4942.957 & 127.4 & 4943.863 & ${ }^{3} \mathrm{~F}_{3}-{ }^{3} \mathrm{P}_{2}$ \\
4988.927 & 59.9 & 4989.954 & ${ }^{3} \mathrm{~F}_{2}-{ }^{3} \mathrm{P}_{0}$ \\
$5159.088^{c}$ & 105.7 & 5160.332 & ${ }^{3} \mathrm{~F}_{3}-{ }^{3} \mathrm{P}_{1}$ \\
$5276.792^{d}$ & 85.0 & 5277.853 & ${ }^{3} \mathrm{~F}_{4}-{ }^{3} \mathrm{P}_{2}$ \\
5721.406 & 533.7 & 5722.297 & ${ }^{3} \mathrm{~F}_{2}-{ }^{1} \mathrm{D}_{2}$ \\
$6087.220^{e}$ & 870.9 & 6088.651 & ${ }^{3} \mathrm{~F}_{3}-{ }^{1} \mathrm{D}_{2}$ \\
\hline${ }^{a}$ Units: $10^{-14}$ erg cm ${ }^{-2} \mathrm{~s}^{-1}$. & \\
${ }^{b}$ Blended with N II $\lambda 2143.452$. & \\
${ }^{c}$ Blended with Fe II $\lambda 5160.214$. & \\
${ }^{d}$ Blended with Fe II $\lambda 5277.480$. & \\
${ }^{e}$ Blended with Ca V $\lambda 6088.058$. &
\end{tabular}

derive an estimate of the Fe VII line flux as $19.4 \pm 3.3 \times$ $10^{-14} \mathrm{erg} \mathrm{cm}^{-2} \mathrm{~s}^{-1}$.

The low resolution optical spectra from STIS do not allow line blending to be studied. For this purpose we have analysed high resolution optical spectra obtained with the UVES instrument on the Kueyen telescope of the VLT in 1999 October. These spectra are not simultaneous with the STIS spectra and we note that emission line fluxes have been found to vary in time for RR Tel (Thackeray 1977; Crawford et al. 1999), with low ionization lines becoming weaker and high ionization lines becoming stronger. The 12 month separation of the UVES and STIS spectra would have led to relative changes in emission line fluxes of at most $10 \%$, and the blending of Fe VII lines with $\mathrm{Fe}$ II lines discussed below will be overestimated by at most this amount. 

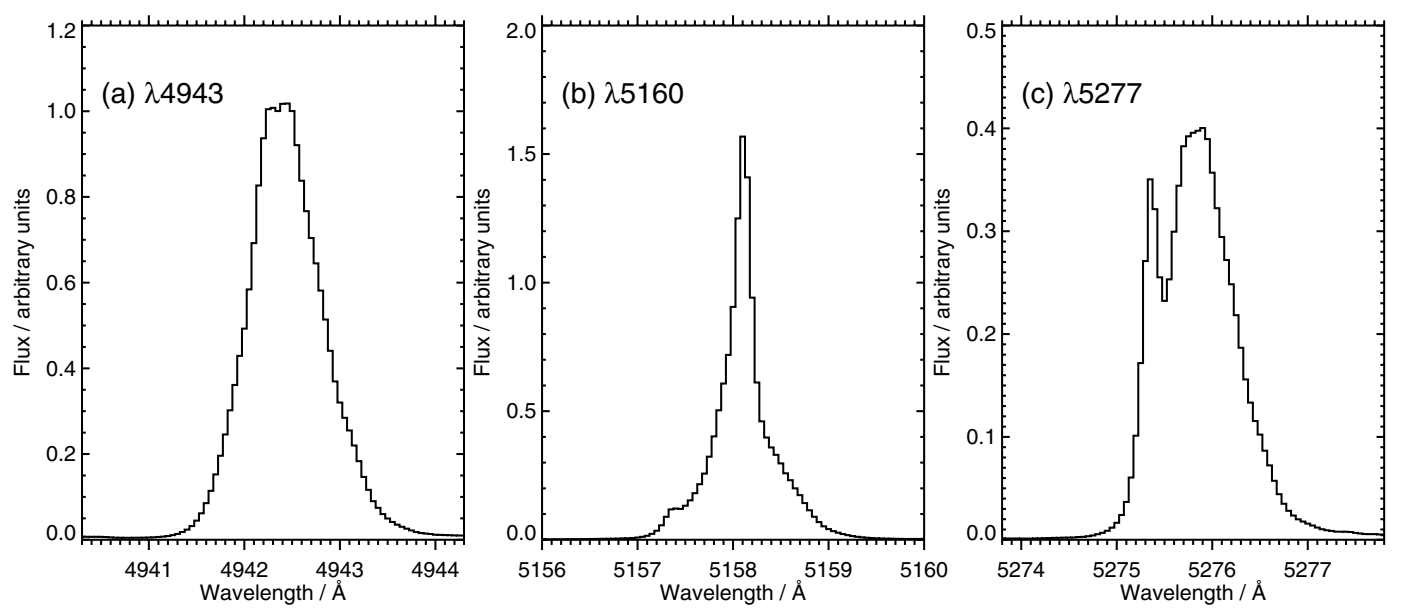

Fig. 3. Spectra from UVES showing three Fe VII lines. The $\lambda 4943$ line is unblended, while both $\lambda 5160$ and $\lambda 5276$ are blended with cooler species.

The UVES spectra were reduced using the MIDAS package by one of us (A. Lobel). The Fe VII optical lines are intrinscially asymmetric (Fig. 3a), and two of the lines clearly demonstrate blending: $\lambda 5160$ and $\lambda 5277$ (Figs. 3b,c). For $\lambda 5160$, we identify the blending line as Fe II $3 \mathrm{~d}^{7} a^{4} \mathrm{~F}_{9 / 2}-3 \mathrm{~d}^{6}\left({ }^{3} \mathrm{H}\right) 4 \mathrm{~s} a^{4} \mathrm{H}_{13 / 2}$ which has a vacuum wavelength of 5160.214 A. Although this is a forbidden transition (and thus has a small $A$-value), it is the only transition from the $a^{4} \mathrm{H}_{13 / 2}$ upper level, and thus gives rise to a strong emission line. The Fe VII $\lambda 4894 / \lambda 5160$ branching ratio suggests a Fe II contribution of $22 \%$, and this is consistent with the appearance of the UVES profile (Fig. 3b). As there are no branching ratios for the $\mathrm{Fe}$ II $a^{4} \mathrm{H}_{13 / 2}$ level then it is not possible to estimate the Fe II contribution to $\lambda 5160$ independently of the Fe VII lines.

The Fe VII $\lambda 5277$ line is blended on the short wavelength side with an Fe II transition that we identify as $3 \mathrm{~d}^{6}\left({ }^{3} \mathrm{G}\right) 4 \mathrm{~s} a^{4} \mathrm{G}_{9 / 2}-3 \mathrm{~d}^{6}\left({ }^{5} \mathrm{D}\right) 4 \mathrm{p} z^{4} \mathrm{~F}_{7 / 2}$, with vacuum wavelength $5277.480 \AA$ A. Another line from this upper level has a wavelength $4557.178 \AA$ and is identified in the STIS spectrum with a flux $5.5 \pm 0.7 \times 10^{-14} \mathrm{erg} \mathrm{cm}^{-2} \mathrm{~s}^{-1}$. The branching ratio (using radiative decay rates from the CHIANTI database) implies that the $\lambda 5277.480$ line has a flux $7.4 \pm 0.9 \times 10^{-14}$, thus contributing $8.7 \%$ to the blended STIS feature. This is consistent with the high resolution UVES spectrum (Fig. 3c).

Selvelli \& Bonifacio (2001) list the Fe VII $\lambda 3587$ and $\lambda 6087$ lines as blended with Fe VI and Ca V lines, respectively. The blending Fe VI line (corresponding to ground configuration transition ${ }^{4} \mathrm{P}_{1 / 2}-{ }^{2} \mathrm{~F}_{5 / 2}$ ) has vacuum wavelength $3588.680 \AA$, i.e., $1.339 \AA$ from the Fe VII line, and so would be easily resolvable from the Fe VII line in the high resolution UVES spectra, yet it is not seen. Further we note that the Fe VI transition shares a common upper level with the $2145.756 \AA$ transition (decays to ${ }^{4} \mathrm{~F}_{3 / 2}$ ), and the branching ratio $\lambda 3588 / \lambda 2145$ is $2.5 \times 10^{-3}$ using the radiative decay rates from CHIANTI. The $\lambda 2145$ line is found in the STIS spectra with a flux $4 \times 10^{-14} \mathrm{erg} \mathrm{cm}^{-2} \mathrm{~s}^{-1}$, implying a $\lambda 3588$ flux of $1 \times 10^{-16} \mathrm{erg} \mathrm{cm}^{-2} \mathrm{~s}^{-1}$ and thus it makes no significant contribution to the Fe VII $\lambda 3587$ line.

The $\mathrm{CaV}$ line is the ${ }^{3} \mathrm{P}_{1}-{ }^{1} \mathrm{D}_{2}$ ground configuration transition with vacuum wavelength $6088.058 \AA$ and is thus $0.593 \AA$ shortward of the Fe VII line. The large width of the feature at this wavelength means the two lines would be blended. Another Ca V line at $5309.580 \AA$ shares the ${ }^{1} \mathrm{D}_{2}$ upper level and the theoretical ratio is 0.19 (using radiative decay rates from the NIST database). The 15309 line is found in the STIS spectra with a flux $7.94 \times 10^{-13} \mathrm{erg} \mathrm{cm}^{-2} \mathrm{~s}^{-1}$, thus implying a $\lambda 6088$ flux of $1.51 \times 10^{-13} \mathrm{erg} \mathrm{cm}^{-2} \mathrm{~s}^{-1}-$ only a $1.7 \%$ contribution to the $\lambda 6087$ feature in the STIS spectrum. Correcting for this leads to a flux for the Fe VII component of $8.56 \times 10^{-12} \mathrm{erg} \mathrm{cm}^{-2} \mathrm{~s}^{-1}$.

The high resolution of the STIS/MAMA spectra allow line widths to be measured, and the $\lambda 2016$ line has a width of $53 \mathrm{~km} \mathrm{~s}^{-1}(0.357 \AA)$. A correlation between line width and ionization potential has been noted before in RR Tel (Penston et al. 1983). The ionization potential of $\mathrm{Fe}^{+6}$ is $99.1 \mathrm{eV}$, and the $\lambda 2016$ line width compares well with the widths of lines of $\mathrm{Ne}^{+4}(97.1 \mathrm{eV})$ and $\mathrm{Mg}^{+4}(109.3 \mathrm{eV})$ : the $\mathrm{Ne} \mathrm{V} \lambda 1574$ line has a width of $46 \mathrm{~km} \mathrm{~s}^{-1}$, and the $\mathrm{MgV} \lambda 1324$ line has a width of $50 \mathrm{~km} \mathrm{~s}^{-1}$, both measured from the STIS spectra.

Figure 4 shows part of the optical spectrum, with the Fe VII and several other prominent lines identified.

\section{Comparison with observations}

\subsection{Branching ratios}

For emission lines that share a common upper level in the ion, the line ratio is insensitive to the plasma conditions in an optically thin plasma and is proportional to the ratio of radiative decay rates for the transitions. Such ratios thus provide a means for checking the quality of radiative data for an ion. Seven such ratios are found in the STIS spectra of RR Tel, and are given in Table 3. Branching ratios in astrophysical spectra are affected by interstellar extinction - wavelength dependent absorption and scattering by interstellar dust grains - usually quantified by the quantity $E(B-V)$. A value of 0 corresponds to no extinction, while 0.10 is the value typically assumed for RR Tel (e.g., Penston et al. 1983). Table 3 thus gives the theoretical ratio values calculated for these two extinction values, using the extinction curve parameterisation of Fitzpatrick (1999). 


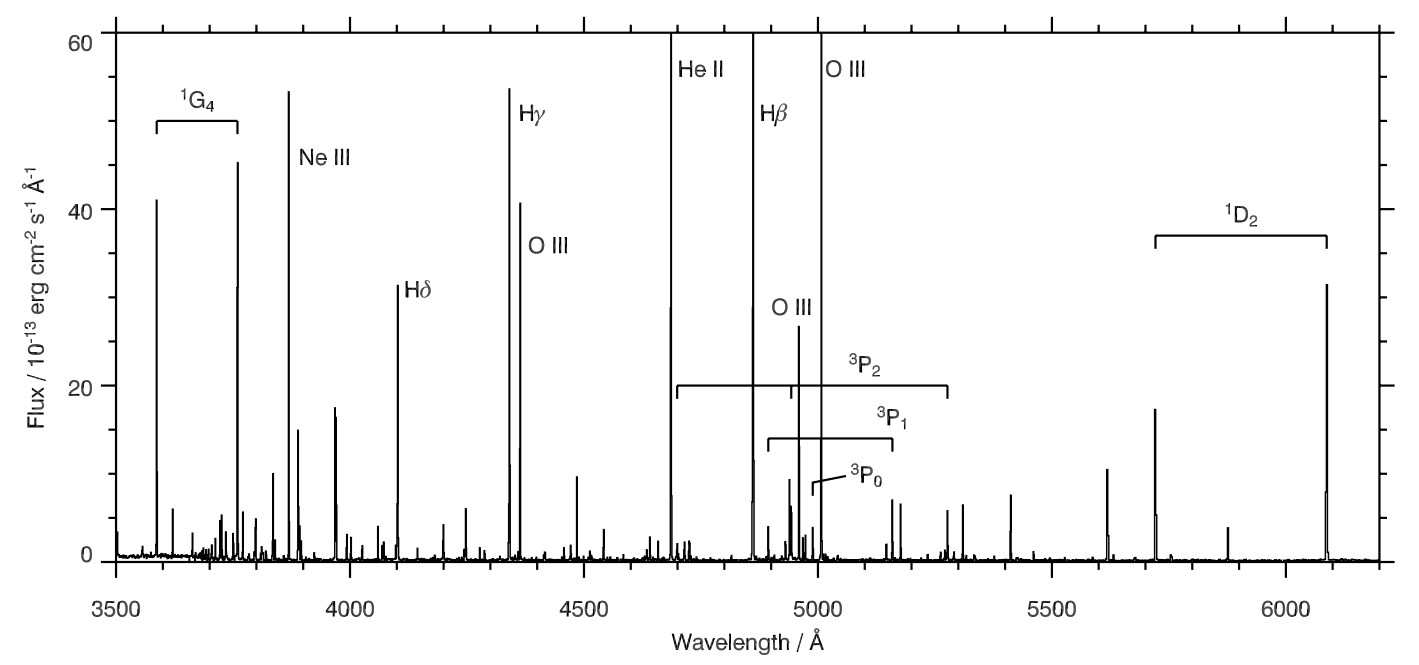

Fig. 4. A section of the optical spectrum of RR Tel from HST/STIS, covering 3500-6200 A. The ten Fe VII lines are identified by their upper emitting level

Table 3. Fe VII branching ratios with observed values from the RR Tel spectrum. Two theoretical values are given: one for no interstellar extinction; the other for an extinction $E(B-V)=0.10$. $\triangleleft$ denotes that the ratio is discrepant with theory by $>1 \sigma ; \triangleleft$ a discrepancy of $>3 \sigma$.

\begin{tabular}{lllll}
\hline \hline \multirow{2}{*}{$\begin{array}{l}\text { Upper } \\
\text { level }\end{array}$} & Ratio & Observed & \multicolumn{2}{c}{$E(B-V)$} \\
\cline { 4 - 5 }${ }^{1} \mathrm{~S}_{0}$ & $\lambda 2143^{a} / \lambda 2016$ & $0.288 \pm 0.054$ & 0.242 & $0.224 \triangleleft$ \\
& $\lambda 2183 / \lambda 2016$ & $0.036 \pm 0.007$ & 0.038 & 0.036 \\
${ }^{1} \mathrm{G}_{4}$ & $\lambda 3587 / \lambda 3759$ & $0.663 \pm 0.047$ & 0.715 & 0.704 \\
${ }^{3} \mathrm{P}_{2}$ & $\lambda 4699 / \lambda 4943$ & $0.247 \pm 0.017$ & 0.226 & $0.221 \triangleleft$ \\
& $\lambda 5277^{b} / \lambda 4943$ & $0.609 \pm 0.045$ & 0.988 & $1.017 \triangleleft$ \\
${ }^{3} \mathrm{P}_{1}$ & $\lambda 4894 / \lambda 5160^{c}$ & $0.553 \pm 0.039$ & 0.695 & $0.678 \triangleleft$ \\
${ }^{1} \mathrm{D}_{2}$ & $\lambda 5722 / \lambda 6088^{d}$ & $0.623 \pm 0.044$ & 0.656 & 0.642 \\
\hline
\end{tabular}

${ }^{a}$ Flux corrected for blend with $\mathrm{N}$ II $\lambda 2143.452$.

${ }^{b}$ Flux corrected for blend with Fe II $\lambda 5277.480$.

${ }^{c}$ Blended with Fe II $\lambda 5160.214$ (not corrected).

${ }^{d}$ Flux corrected for blend with $\mathrm{Ca}$ V $\lambda$ 6088.058.

Only three of the seven ratios agree with the theoretical values within the $1 \sigma$ error bars on the fluxes. Of the discrepant ratios, $\lambda 4894 / \lambda 5160$ is affected by the blend to the $\lambda 5160$ line mentioned in the previous section which accounts for the observed ratio exceeding the theoretical value. The discrepancy for the $\lambda 5277 / \lambda 4943$ ratio can not be resolved by blending in the $\lambda 4943$ line as this would then make the $\lambda 4699 / \lambda 4943$ disagree with theory. Future calculations of radiative decay rates should aim to resolve the problems highlighted here.

\subsection{Derivation of physical parameters}

For an optically thin plasma the line ratios amongst the various emission lines are determined entirely by the plasma temperature and density, and interstellar extinction. From the STIS fluxes we can thus determine each of these parameters. Rather than consider only individual line ratios as is often done, we instead employ a minimization procedure to all of the observed emission lines simultaneously. This provides a much more stringent test on the atomic data, but gives greater confidence in the derived parameters.

The method applied is as follows: lines from the same upper level are summed, thus giving six independent measured quantities corresponding to the six upper emitting levels (see Table 2). The $\lambda 2143, \lambda 5277$ and $\lambda 6087$ line fluxes have been corrected for line blending following the discussion in Sect. 3, but the $\lambda 5160$ is not included in the analysis as the blending contribution can not be estimated independently of the $\lambda 4894 / \lambda 5160$ branching ratio. The summed line fluxes for each level are then divided through by the summed flux for the ${ }^{1} \mathrm{D}_{2}$ level, leading to five independent measurables.

A least-squares minimization procedure was applied using the MPFIT package such that the temperature is allowed to vary over the range $3.9 \leq \log T / \mathrm{K} \leq 5.5$, the electron density over $5.0 \leq \log N_{\mathrm{e}} / \mathrm{cm}^{-3} \leq 9.0$, and the extinction $0.0 \leq E(B-V) \leq 1.0$. At each iteration, the level balance equations for $\mathrm{Fe}$ VII are solved at the temperature and density values using the CHIANTI software, and emissivities for each 
Table 4. Comparison of observed line ratios with those derived from the fitting procedure. The ratios are formed by summing lines from a common upper level, and comparing with the summed lines from the ${ }^{1} \mathrm{D}_{2}$ level.

\begin{tabular}{lll}
\hline \hline \multirow{2}{*}{$\begin{array}{l}\text { Upper } \\
\text { level }\end{array}$} & \multicolumn{2}{c}{ Ratio } \\
\cline { 2 - 3 } & Observed & Fitted \\
\hline${ }^{3} \mathrm{P}_{0}$ & $0.043 \pm 0.003$ & 0.039 \\
${ }^{3} \mathrm{P}_{1}$ & $0.042 \pm 0.003$ & 0.042 \\
${ }^{3} \mathrm{P}_{2}$ & $0.170 \pm 0.008$ & 0.183 \\
${ }^{1} \mathrm{G}_{4}$ & $1.003 \pm 0.051$ & 1.016 \\
${ }^{1} \mathrm{~S}_{0}$ & $0.064 \pm 0.005$ & 0.063 \\
\hline
\end{tabular}

line calculated. These are then adjusted for the extinction value, and line ratios compared with the observed values.

The final results of the minimization procedure were $\log T / \mathrm{K}=4.50 \pm 0.23, \log N_{\mathrm{e}} / \mathrm{cm}^{-3}=7.25 \pm 0.05$ and $E(B-V)=0.11 \pm 0.16$. The $\chi^{2}$ value was 2.3 . A comparison between the observed ratios and the fitted values is given in Table 4. Agreement is good, with three ratios lying within the error bars on the data. The discrepancy for the ${ }^{3} \mathrm{P}_{2}$ level is likely due to the branching ratio problem for the ${ }^{3} \mathrm{P}_{2}$ level noted in Sect. 4.1.

The $E(B-V)$ value is poorly constrained due to the fact that low values of the extinction cause relatively little change in line fluxes even over the wide wavelength coverage of the Fe VII lines. Performing the minimization with $E(B-V)$ fixed at 0.10 gives temperature and density values of $\log T / \mathrm{K}=$ $4.50 \pm 0.02, \log N_{\mathrm{e}} / \mathrm{cm}^{-3}=7.25 \pm 0.05$.

This same minimization procedure was repeated using the previous CHIANTI dataset, but the solution did not converge within the prescribed parameter bounds, demonstrating that the Keenan \& Norrington (1987) data does not accurately reproduce the observed fluxes.

Previous estimates of the RR Tel nebula temperature through emission line diagnostics give values of $10-25000 \mathrm{~K}$ (Penston et al. 1983; Hayes \& Nussbaumer 1986; Doschek \& Feibelmann 1993; Espey et al. 1996), consistent with the Fe VII result within the error bars.

Densities previously derived for the RR Tel nebula vary widely with ionization species, as best illustrated by Table 6 of Hayes \& Nussbaumer (1986), where values $4.9 \leq$ $\log N_{\mathrm{e}} / \mathrm{cm}^{-3} \leq 9.0$ are given. All the species studied there, however, are low ionization and so not comparable to Fe VII. Espey et al. (1996) adopt a density of $\log N_{\mathrm{e}} / \mathrm{cm}^{-3}=6.0$ from an analysis of $\mathrm{Ne} \mathrm{V}$ and $\mathrm{Ne}$ VI lines in spectra from the Hopkins Ultraviolet Telescope (HUT). The ionization potentials of these ions are 97.1 and $126.2 \mathrm{eV}$, compared to $99.1 \mathrm{eV}$ for $\mathrm{Fe}^{+6}$, and so the ions are comparable. We note that the HUT spectra are only of moderate spectral resolution, and high resolution spectra from FUSE would be valuable in improving the density estimate from the Ne VI lines.

Previous estimates for the extinction towards RR Tel include the value of $E(B-V)=0.10$ derived from He II recombination lines in IUE spectra (Penston et al. 1983), and $0.08 \pm 0.03$ from the $\mathrm{NeV} \lambda 2974 / \lambda 1574$ branching ratio
(Espey et al. 1996). This latter pair of lines are found in the STIS spectrum, and we find an observed ratio of $0.469 \pm 0.053$. The theoretical ratio is 0.376 (Galavís et al. 1997), leading to an extinction of $0.109_{-0.059}^{+0.052}$, using the extinction curve parameterisation of Fitzpatrick (1999). This is consistent with the upper limit $(0.27)$ found here from the Fe VII lines.

\section{Conclusions}

The $3 \mathrm{~d}^{2}$ ground configuration transitions of Fe VII provide excellent plasma diagnostic opportunities for nebular plasmas illuminated by hot stars. The most recent Fe VII electron excitation data from Berrington et al. (2000) are a great improvement over the previous data-set of Keenan \& Norrington (1987) and the complete set of Fe VII lines in the HST/STIS spectra of RR Tel yield values for the electron temperature and density, and extinction. The temperature and extinction are consistent with values derived from other ions, while we believe the density estimate is the most accurate obtained so far for RR Tel at ionization levels of around $100 \mathrm{eV}$. Four of the seven Fe VII branching ratios do not agree with the observed values within the error bars. One of these ratios $(\lambda 4894 / \lambda 5160)$ is affected by blending in the $\lambda 5160$ line and the UVES profile for the $\lambda 5160$ is consistent with the blending Fe II line accounting for the blend.

The accurate flux calibration of STIS, coupled with the strong Fe VII lines found in RR Tel, make the STIS RR Tel spectrum discussed here an excellent benchmark for checking the accuracy of atomic physics calculations for Fe VII.

The atomic data used here will be included in version 5 of the CHIANTI atomic database.

Acknowledgements. A.L. acknowledges financial support provided by STScI grants HST-GO-09369.01-A and HST-GO-10212.01-A, and by NASA FUSE grants GI-D107 and GI-E068.

\section{References}

Berrington, K. A., Nakazaki, S., \& Norrington, P. H. 2000, A\&AS, 142,313

Dere, K. P., Landi, E., Mason, H. E., Monsignori Fossi, B. C., \& Young, P. R. 1997, A\&AS, 125, 149

Doschek, G. A., \& Feibelmann, W. A. 1993, ApJS, 87, 331

Espey, B., Keenan, F. P., McKenna, F. C., Feibelman, W. A., \& Aggarwal, K. M. 1996, ApJ, 465, 965

Fitzpatrick, E. L. 1999, PASP, 111, 63

Galavís, M. E., Mendoza, C., \& Zeippen, C. J. 1997, A\&AS, 123, 159

Hayes, M. A., \& Nussbaumer, H. 1986, A\&A, 161, 287

Keenan, F. P., \& Norrington, P. H. 1987, A\&A, 181, 370

Jordan, S., Mürset, U., \& Werner, K. 1994, A\&A, 283, 475

Crawford, F. L., Mckenna, F. C., Keenan, F. P., et al. 1999, A\&AS, 139,135

Nussbaumer, H., \& Storey, P. J. 1982, A\&A, 113, 2

Penston, M. V., Benvenuti, P., Cassatella, A., et al. 1983, MNRAS, 202, 833

Selvelli, P. L., \& Bonifacio, P. 2001, A\&A, 364, L1

Thackeray, A. D. 1977, Mem. RAS, 83, 1

Träbert, E., Calamai, A. G., Gwinner, G., et al. 2003, J. Phys. B, 36, 1129

Young, P. R., Del Zanna, G., Landi, E., et al. 2003, ApJS, 144, 135 